\title{
Laser Treatment of ITO and ZnO Nanoparticles for the Production of Thin Conducting Layers on Transparent Substrates
}

\author{
Marcus BAUM ${ }^{* 1,2}$, Ilya ALEXEEV ${ }^{* 1,2}$ and Michael SCHMIDT ${ }^{* 1,2}$ \\ ${ }^{* 1}$ Chair of Photonic Technologies, Friedrich-Alexander-University Erlangen-Nuremberg, Paul-Gordan-Str. 3, 91052 \\ Erlangen, Germany \\ E-mail:marcus.baum@lpt.uni-erlangen.de \\ ${ }^{* 2}$ Erlangen Graduate School in Advanced Optical Technologies (SAOT), Friedrich-Alexander-University Erlangen- \\ Nuremberg, Paul Gordan Str. 6, 91052 Erlangen, Germany
}

\begin{abstract}
Layers of ITO nanoparticles were deposited on soda-lime substrates by spincoating in order to produce conductive transparent electrodes. Different lasers in the UV and mid infrared wavelength region were used for post deposition treatment of the layers to remove stabilizing agents contained in the layers to enhance particle-particle contact and significantly increase conductivities. Irradiation with lasers in the infrared region showed good results in terms of stabilizer removal without undesired oxidizing effects to the particle layer. Conductivities of up to $120 \mathrm{~S} / \mathrm{cm}$ were achieved, which is comparable to layer treatment by annealing and a subsequent chemical reducing step.
\end{abstract}

DOI:10.2961/jlmn.2011.03.0003

Keywords: ITO, ZnO, nanoparticles, laser melting, transparent, conducting layers

\section{Introduction}

Due to their high transparency in the visible spectral range and their high electrical conductivity, tin-doped indium oxide (ITO) and doped zinc oxide (i.e. Al-doped $\mathrm{ZnO}$ ) offer a wide range of applications in optoelectronic devices, such as OLED- and LC-displays, touch screens or solar cells [1]. For these applications, the required conducting films are usually created by vapor deposition. However, this is a time consuming and costly technique, as vacuum setups are required. A very promising alternative to this process, especially when considering applications for printable and flexible electronics, is the formation of conducting films based upon nanoparticle deposition and subsequent consolidation via laser irradiation. This versatile processing technique offers the possibility of selective spatial and temporal energy deposition.

Up to now, the established way for creating conductive layers from ITO nanoparticles has been the following: Nanoparticles contained in a suspension are coated on the desired substrate by spin coating, dip coating or printing techniques. Subsequent steps are necessary to remove the stabilizer contained in the suspension, which is required to prevent agglomeration of the particles prior to deposition. After stabilizer removal, the conductivity can be increased by further steps like forming gas treatment, which increases the number of oxygen vacancies in the particle material [2]. Also, other techniques like the infiltration of the particular ITO layer by an ITO precursor solution [3] or annealing in different atmospheres [2] have been proposed. Conductivities reached in ITO nanoparticle films so far range from 6 $\mathrm{S} / \mathrm{cm}$ to $132 \mathrm{~S} / \mathrm{cm}[2,3]$.

When it comes to laser melting of particle layers, a contamination-free, i.e. stabilizer-free particle layer is inevitably required in order to obtain good results. In this work, we focus on an efficient way to remove the stabilizer from the particle layer by laser irradiation and compare it to the usage of annealing and subsequent forming gas treatment.

\section{Experimental part}

In our experiments, we used ethanol-based suspensions of either ITO or undoped $\mathrm{ZnO}$ particles, commercially available from Evonik Degussa $\mathrm{GmbH}$, with particle sizes ranging from $20 \mathrm{~nm}$ to $120 \mathrm{~nm}$. $20 \%$ wt of the particles are dispersed in Ethanol. Agglomeration of the particles in the suspensions was prevented by addition of 2-[2-(2Methoxyethoxy)ethoxy]acetic acid, also known as trioxydecanoic acid (TODS), a stabilizer which turned out to be the best choice in terms of particle film formation and long term stability of particle suspensions [4]. Layers of $700 \pm 40 \mathrm{~nm}$ in thickness were produced by spin coating of the particle suspensions on soda-lime substrates. The film thickness was measured by a DekTak profilometer.

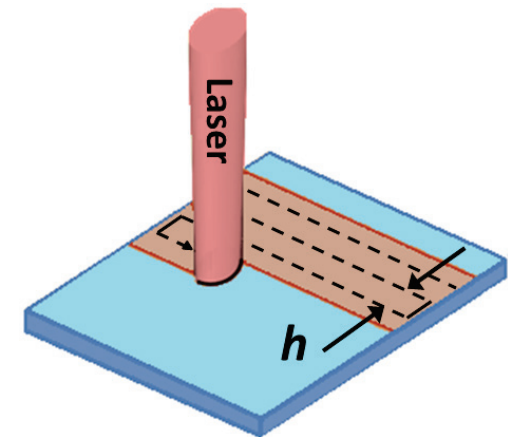

Fig. 1. Schematic diagram of the experimental setup: The laser beam at normal incidence to the sample surface is scanned linewisely over the entire sample maintaining the hatch distance $h$ between the lines. 
For the laser treatment of ITO films we used a Wavelight Glissando Er:YAG laser with a wavelength of $2940 \mathrm{~nm}$, a pulse length of $350 \mu \mathrm{s}$ and a maximum output power of 20 $\mathrm{W}$, while the repetition rate ranged from $1 \mathrm{~Hz}$ to $20 \mathrm{~Hz}$. The laser spot on the sample was $4 \mathrm{~mm}$ in diameter. While the laser was kept at a constant distance and the laser beam at normal angle to the sample surface, the sample was moved by two linear stages in order to irradiate the whole sample surface. The hatch distance $h$ i.e. the distance between two parallel laser lines was usually chosen slightly smaller than the laser spot diameter to ensure complete coverage. It was varied between 1 and $3 \mathrm{~mm}$. Fig. 1 shows a schematic diagram of the experimental setup.

For comparison purposes an identical sample was annealed on a hot plate at $450{ }^{\circ} \mathrm{C}$ for $30 \mathrm{~min}$ and subsequently treated by forming gas $\left(95 \% \mathrm{~N}_{2}, 5 \% \mathrm{H}_{2}\right)$ at $300^{\circ} \mathrm{C}$ for 30 minutes.

The transmission and reflection properties of the samples and substrate were measured by an UV-VIS-NIR spectrometer between $200 \mathrm{~nm}$ and $2700 \mathrm{~nm}$. In the region of longer wavelengths an FTIR spectrometer was used. The Sheet resistances were acquired by a linear 4-point setup. Post treatment was performed by a Lambda Physik LPX $315 \mathrm{i} \mathrm{KrF}$ excimer laser with a wavelength of $248 \mathrm{~nm}$ and a pulse length of $30 \mathrm{~ns}$ at a repetition rate of $1 \mathrm{~Hz}$ to $100 \mathrm{~Hz}$, a maximum output power of $80 \mathrm{~W}$ and a raw beam size of $30 \mathrm{~mm} \times 15 \mathrm{~mm}$. Due to the vast beam area, no scanning was performed on the samples.

\section{Results and discussion}

The characteristic feature of transparent electrodes in addition to low resistivity is high transmission in the visible range. The transmission curves of an as deposited ITO particle layer on a soda lime substrate and the transmission of the soda lime substrate itself, measured by an UV-VIS-NIR spectrometer between $200 \mathrm{~nm}$ and $2700 \mathrm{~nm}$ are shown in fig. 2a). The untreated ITO layer shows the typical behaviour of a heavily doped semiconductor. Starting at zero, the transmission increases to almost hundred percent above $320 \mathrm{~nm}$, where the band gap is located. It is worth mentioning that the onset in transmission of the soda lime substrate at almost the same wavelength is just a coincidence and did not influence the transmission measurements of the layer. This was confirmed by a measurement of a comparable particle layer on a fused silica substrate, which is transparent in this wavelength regime. Due to the high amount of free charge carriers in the ITO particles, the transmission shows a decrease starting slightly above $1000 \mathrm{~nm}$. Here, the incident photons can interact with the free electrons contained in the material. This downturn in transmission is also called plasma edge. According to the Drude model, which considers free electrons, the so-called plasma frequency $\omega_{p}$ is given by $\omega_{p}^{2}=n e^{2} / \varepsilon_{0} m_{e f f}$ where $n$ represents the charge carrier density and $m_{e f f}$ is the effective electron mass. The charge carrier density can roughly be estimated by inserting values for $\omega_{p}$, which can be calculated from $\lambda_{\mathrm{p}}$ $=1450 \mathrm{~nm}$, the wavelength at which the transmission shows the highest gradient, and $m_{e f f}=0.4 m_{e}$, which has been determined experimentally for similar material [5]. This leads to $n \approx 2 \cdot 10^{20} / \mathrm{cm}^{3}$, which is in good agreement with experimental data obtained by other groups [6]. The
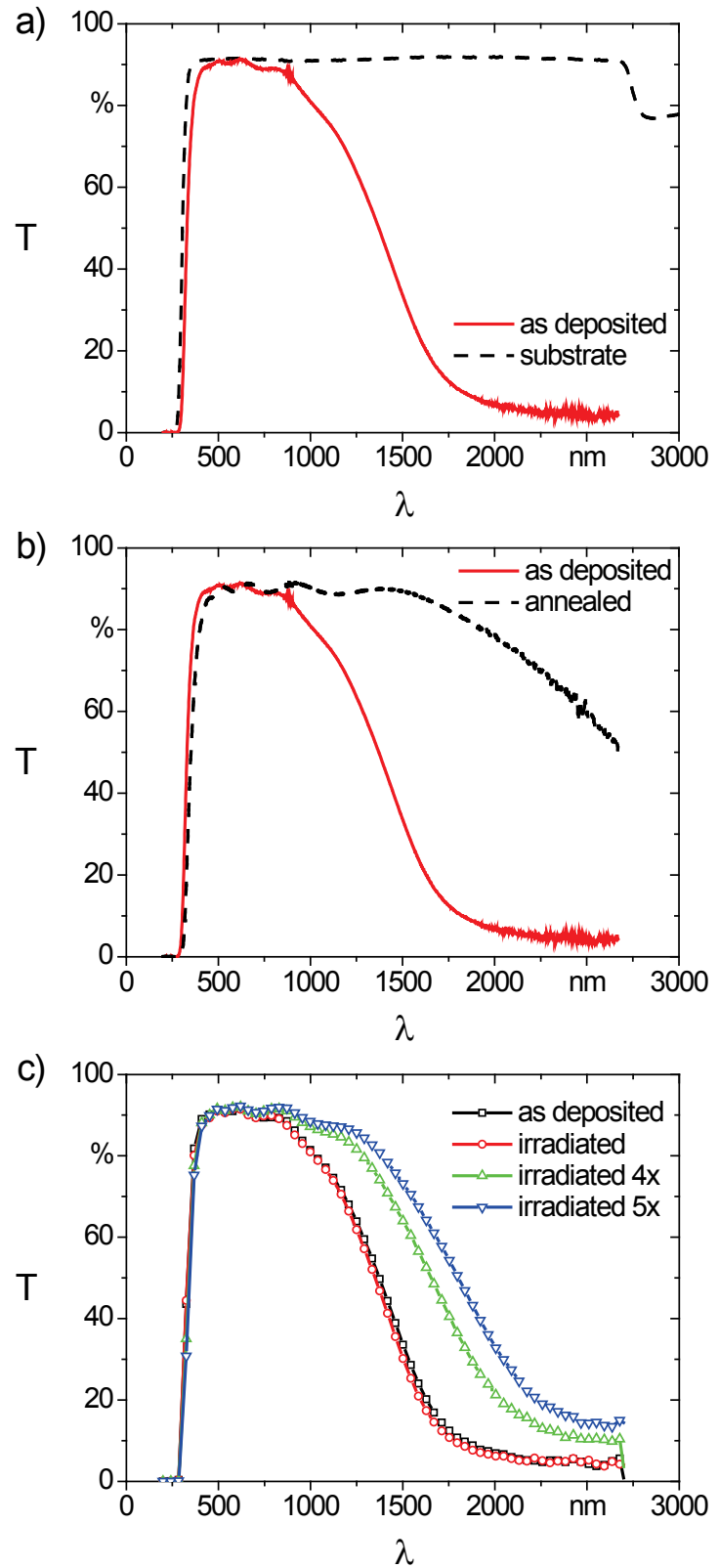

Fig. 2. a) Transmission spectra of the soda-lime substrate and an untreated ITO particle layer on soda-lime glass. b) Transmission spectra of an untreated ITO particle layer on soda lime glass and the same layer after annealing at $450{ }^{\circ} \mathrm{C}$ for 30 min. c) Transmission spectra of an untreated ITO particle layer and the same layer after single, fourfold and fivefold irradiation by Er:YAG at $10 \mathrm{~Hz}, 600 \mathrm{~mJ}$

$\mathrm{ZnO}$ particle layer (not shown here), in comparison, shows a similar transmission curve in the UV and visible wavelength region, while it does not show a drop of transmission in the infrared regime up to $2700 \mathrm{~nm}$, as it features a much lower electron density compared to ITO due to the lack of doping.

For transparent electrode applications, the most important particle layer parameter besides transparency in the visible wavelength regime is its conductivity. Conductivity of ITO due to the presence of free electrons stems from doping by tin, as well as from oxygen defect sites, which both act as electron donors. In particle layers, it could also be increased by sintering or melting of the 


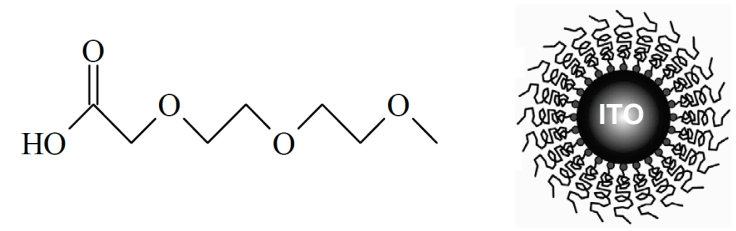

Fig. 3. Left: structural formula of TODS. Right: sketch of a particle functionalized by TODS

particles which enlarges the particle-particle boundary area in comparison to loosely touching particles. After deposition, our films show an initial sheet resistance of approximately $1 \mathrm{M} \Omega$ (which equals to a conductivity in the order of $1 \cdot 10^{-2} \mathrm{~S} / \mathrm{cm}$ ) and thus are not yet of use for electronic applications. It is important to note that the initially very high sheet resistance in the $M \Omega$ regime of the particle layers after deposition is due to the functionalization of the particels by TODS (fig.3). This stabilizing agent leads to very high long term stability of the particle suspensions and also provides smooth particle film formation. However, it is less beneficial when it comes to conductivity, as every single particle is coated by a non-conductive layer of approximately $1 \mathrm{~nm}$ in thickness (fig.3). Consequently, in order to increase conductivity after deposition, it is necessary to remove the stabilizing agent from the particle layer. Usually, this is achieved by annealing of the samples in air for about 30 minutes at temperatures ranging from $300{ }^{\circ} \mathrm{C}$ to $550{ }^{\circ} \mathrm{C}$ subsequent to the deposition, leading to degeneration and evaporation of the stabilizer $[2,6]$. Conductivities on the order of $1 \mathrm{~S} / \mathrm{cm}$ are obtained after the annealing step caused by the formation of particle-particle contact. However, there is a major drawback in this approach besides the long processing time. Fig $2 \mathrm{~b}$ ) shows the transmission spectra of the ITO particle film after deposition and after annealing in air at $450^{\circ} \mathrm{C}$ for 30 minutes. It is clearly visible that after annealing the transmission window increases, i.e. the plasma edge moves towards longer wavelengths indicating a depletion of free charge carriers. In addition, a slight shift of the band gap towards longer wavelengths denotes a reduction of the total number of free charge carriers according to the Burstein-Moss effect. The depletion of free charge carriers can be explained by oxidization of the material and thus reduction of the number of oxygen vacancies resulting in less free electrons. Subsequent treatment of the same layer by forming gas $\left(95 \% \mathrm{~N}_{2}, 5 \%\right.$ $\mathrm{H}_{2}$ ) at $300^{\circ} \mathrm{C}$ for 30 minutes can provide chemical reduction of the particle layer and therefore reintroduce oxygen vacancies, which were removed during annealing in air. The transmission spectrum (not shown here) reaches almost its initial shape. Conductivities of $110 \pm 15 \mathrm{~S} / \mathrm{cm}$ were reached in our experiment.

Laser treatment of the particle layers can provide a promising alternative to the conventional procedure. Since the particle layers show high transmission in the visible wavelength range, obviously only lasers in the UV, i.e. below $320 \mathrm{~nm}$, or lasers in the near infrared, i.e. above $1500 \mathrm{~nm}$ come into consideration for the laser treatment of a particle layer. Thus, different lasers in the UV and infrared wavelength region were used for treatment. The results of two of them are shown in the following section.
In the UV region, a $\mathrm{KrF}$ excimer laser with a wavelength of $248 \mathrm{~nm}$ was used. However, due to the efficient absorption and low penetration depth in ITO [7] and $\mathrm{ZnO}$, irradiaton at this wavelength leads to highly localized energy deposition in the surface region of the particle layer and hence does heat up only some tens of nanometers of the particle layer. Therefore, the stabilizer can only be evaporated on the film surface. High pulse energies lead to degeneration and impulsive evaporation of the stabilizer, which in its turn causes either loss of transparency due to carbonization of the stabilizer or ablation of the layer. Thus, a laser with a near infrared wavelength has been chosen for layer treatment. It is important to mention that this wavelength region is not suitable for undoped $\mathrm{ZnO}$ particle layers because here, the onset of absorption or the decrease in transmission respectively takes place at longer wavelengths due to the lower content of free charge carriers, i.e. undoped $\mathrm{ZnO}$ is still transparent in the near infrared and is not significantly affected by light in this wavelength region.

For the laser treatment of ITO films we used an Er:YAG laser with a wavelength of $2940 \mathrm{~nm}$. Measurements of transmission and reflection at the laser wavelength with the laser itself and utilizing a FTIR spectrometer were carried out on both the as deposited ITO particle layer as well as the laser irradiated stabilizer-free particle layer. The absorption of about $51 \%$ of the incident light could be determined independently of the presence of the stabilizer, which gives rise to the conclusion that the main part of the irradiated light is absorbed in the particles themselves and not in the surrounding stabilizer. A laser penetration depth of approximately $1 \mu \mathrm{m}$ could be estimated. Consequently, the laser irradiation affects the whole layer in depth. Irradiation leads to an efficient and localized heating of the particles in a very short period of time and thus, to the degeneration and evaporation of the stabilizer located on the particle surfaces.

The optimal parameters for stabilizer removal from the particle layer in air are a pulse energy of $600 \mathrm{~mJ}$ at a repetition rate of $10 \mathrm{~Hz}$ with a scanning speed of $20 \mathrm{~mm} / \mathrm{s}$ and a hatch distance $h$ of $3 \mathrm{~mm}$, leading to a laser spot overlap of about $50 \%$ and a scanning time of about $10 \mathrm{~s}$ for a sample size of $25 \mathrm{~mm} \times 25 \mathrm{~mm}$. In the following the treatment of a sample with these parameters is referred to as irradiation. With lower pulse energies no or only unsatisfying stabilizer removal could be achieved, which can easily be determined via measurement of the sheet resistance. At energies above $800 \mathrm{~mJ}$, the glass substrate started to form cracks at the surface caused by the high heating and cooling rates. With the optimal laser settings, sheet resistances as low as $125 \mathrm{Ohm} / \mathrm{sq}$ were achieved, which, at a layer thickness of $660 \mathrm{~nm}$, correspond to a conductivity of about $120 \mathrm{~S} / \mathrm{cm}$ and hence are comparable to particle layers prepared by annealing and a subsequent forming gas treatment. Fig 2c) shows the transmission curve of an ITO particle layer of about $700 \mathrm{~nm}$ in thickness right after deposition (black curve). It turns out that the transmission curve of the same layer after laser irradiation (shown in red) has the very same shape i.e. it does not show a shift in the plasma edge. This indicates that no oxidization took place during treatment. Consequently, in comparison to the common annealing step, a subsequent reduction step by forming gas treatment is not necessary. 


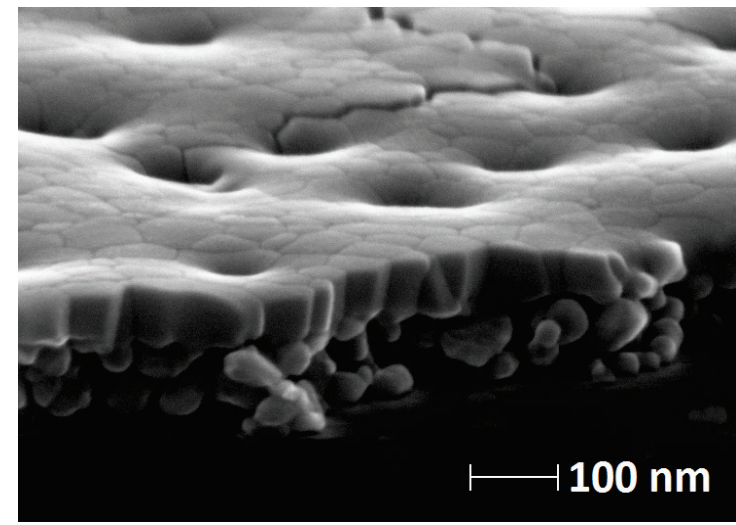

Fig. 4. SEM micrograph of a cross-section of an Excimerlaser irradiated ITO particle layer. For better image quality, the film was prepared on an $\mathrm{Si} / \mathrm{SiO} 2$ substrate.

The green and blue curves show the transmission of the same layer after several irradiations (4 and 5 times). A shift in the plasma edge is clearly observable, indicating gradual oxidization of the ITO layer. In addition, a decrease in conductivity to about half of its value after the first irradiation is observed. Although the experiments so far were carried out only on ITO particle layers, we expect them to work equally on doped $\mathrm{ZnO}$ particle layers due to similar optical properties.

The pure particulate layers can now be treated further in order to achieve higher levels of consolidation and thus higher conductivities resulting from increased contact areas between the particles. The most straight forward approach would be to use the same Er:YAG laser which was also used for the stabilizer removal at higher pulse energies. However, this leads to a new problem. Since both ITO and $\mathrm{ZnO}$ feature melting temperatures as high as $2000 \mathrm{~K}$ and the penetration depth of the laser radiation is approximately $1 \mu \mathrm{m}$, as mentioned above, higher energies per pulse lead to significant thermal stress of the underlying substrate. In our experiments this inevitably leads to cracking or melting of the glass substrate's surface induced by high thermal gradients and temperatures, not only for soda-lime glass, but also for more durable substrates as fused silica or sapphire glass. Thus, in order to protect the substrate, lasers in the UV wavelength regime below $320 \mathrm{~nm}$ should be used, as these wavelengths are very well absorbed on account of the band gap of ITO and $\mathrm{ZnO}$. Thus, they show low penetration depths in the order of $50 \mathrm{~nm}$ in bulk material [7] resulting in lower thermal stress to the substrate. We are currently working with a Lambda Physik LPX 315i KrF Excimer laser (properties mentioned above).

First experiments showed that laser irradiation can indeed lead to consolidation of the particle layer and therefore, it can increase the layer density significantly without harming the substrate. Fig. 4 shows a cross-section of an excimer laser irradiated ITO particle layer. It can be observed that a consolidation of the top layer took place within approximately $50 \mathrm{~nm}$, which corresponds to the laser penetration depth.

\section{Conclusion}

In this work, we have shown the applicability of lasers for the treatment of transparent conductive oxide particle layers. Particle layer preparation i.e. removal of the stabilizer could be achieved by Er:YAG laser irradiation in one step without hot plate treatment and subsequent steps like chemical reduction of the layer. Conductivities of up to $120 \mathrm{~S} / \mathrm{cm}$ were achieved. Thus, for small samples laser treatment offers great advantages in terms of time-saving compared to the common procedure of layer preparation by annealing and subsequent post treatment steps. Furthermore, stabilizer removal can be achieved selectively in space, which can be valuable especially for printed electronics applications. Moreover, UV-laser treatment was proposed for further consolidation of the particle layers. The first experiments have shown very promising results for particle consolidation, while at the same time the substrate is protected from thermal damage.

\section{Acknowledgements}

The support of the Deutsche Forschungsgemeinschaft (Graduiertenkolleg 1161/2) is gratefully acknowledged. Additionally, we are grateful for the generous support by Evonik Degussa GmbH. Moreover, the authors gratefully acknowledge funding of the Erlangen Graduate School in Advanced Optical Technologies (SAOT) by the German Research Foundation (DFG) in the framework of the German excellence initiative.

\section{References}

[1] M. Groß, Druckbare, nanopartikuläre Indiumzinnoxidschichten für optoelektronische Anwendungen, dissertation Friedrich-AlexanderUniversity Erlangen-Nuremberg (2009)

[2] G. Guenther, G. Schierning, R. Theissmann, R. Kruk, R. Schmechel, C Baehtz, A. Prodi-Schwab, J. Appl. Phys. 104 (2008)

[3] M. Gross, N. Linse, I. Maksimenko, P.J. Wellmann, Adv. Eng. Mat 295 (2009)

[4] A. Reindl, Dispersing and Stabilizing Semiconducting Nanoparticles for Application in Printable Electronics, dissertation Friedrich-AlexanderUniversity Erlangen-Nuremberg (2009)

[5] I. Hamberg, C.G.Granqvist: J. Appl. Phys. 60, R123 (1986)

[6] J. Ederth, A. Hultaker, G.A. Niklasson, P. Heszler, A.R. van Doorn, M.J. Jongerius, D. Burgard, C.G. Granqvist, Appl. Phys., A 81, $1363-$ $1368,(2005)$

[7] G. Legeay, X. Castel, R. Benzerga, J. Pinel, Phys. stat. sol. (c) 3250 (2008)

(Received: June 03, 2011, Accepted: October 22, 2011) 